Aslak Tveito · Ragnar Winter

\title{
Introduction to Partial Differential Equations
}

A Computational Approach

算 Springer 


\section{Contents}

1 Setting the Scene 1

1.1 What Is a Differential Equation? . . . . . . . . . 1

1.1.1 Concepts . . . . . . . . . . . . . 2

1.2 The Solution and Its Properties . . . . . . . . . . . . 4

1.2.1 An Ordinary Differential Equation . . . . . . . . 4

1.3 A Numerical Method . . . . . . . . . . . . . . . 6

1.4 Cauchy Problems . . . . . . . . . . . . . . . . . 10

1.4.1 First-Order Homogeneous Equations . . . . . . . 11

1.4.2 First-Order Nonhomogeneous Equations . . . . . . . 14

1.4.3 The Wave Equation . . . . . . . . . . . 15

1.4 .4 The Heat Equation . . . . . . . . . . . . 18

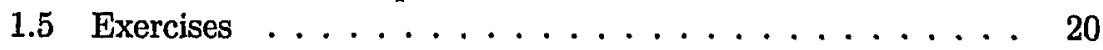

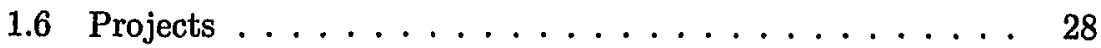

2 Two-Point Boundary Value Problems 39

2.1 Poisson's Equation in One Dimension . . . . . . . . . 40

2.1 .1 Green's Function . . . . . . . . . . . . . 42

2.1.2 Smoothness of the Solution . . . . . . . . . 43

2.1.3 A Maximum Principle . . . . . . . . . . . . . 44

2.2 A Finite Difference Approximation . . . . . . . . . . . 45

2.2 .1 Taylor Series . . . . . . . . . . . . . 46

2.2.2 A System of Algebraic Equations . . . . . . . . . 47

2.2.3 Gaussian Elimination for Tridiagonal Linear Systems $\quad 50$

2.2.4 Diagonal Dominant Matrices . . . . . . . . . . 53 
2.2.5 Positive Definite Matrices . . . . . . . . . . . . 55

2.3 Continuous and Discrete Solutions . . . . . . . . . 57

2.3.1 Difference and Differential Equations . . . . . . . 57

2.3.2 Symmetry . . . . . . . . . . . . . . 58

2.3.3 Uniqueness . . . . . . . . . . . . . . 6 61

2.3.4 A Maximum Principle for the Discrete Problem . . . 61

2.3.5 Convergence of the Discrete Solutions ... . . . 63

2.4 Eigenvalue Problems . . . . . . . . . . . . . . 65

2.4.1 The Continuous Eigenvalue Problem . . . . . . . 65

2.4.2 The Discrete Eigenvalue Problem . . . . . . . . . . . 68

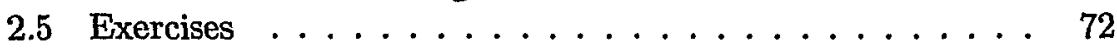

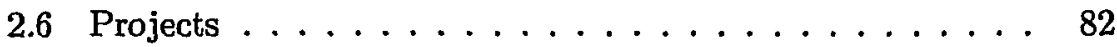

3 The Heat Equation $\quad \mathbf{8 7}$

3.1 A Brief Overview . . . . . . . . . . . . . . . 88

3.2 Separation of Variables . . . . . . . . . . . . 90

3.3 The Principle of Superposition . . . . . . . . . . . 92

3.4 Fourier Coefficients . . . . . . . . . . . . . 95

3.5 Other Boundary Conditions . . . . . . . . . . . . . . 97

3.6 The Neumann Problem . . . . . . . . . . . . . . . . 98

3.6.1 The Eigenvalue Problem . . . . . . . . . . . . . . 99

3.6.2 Particular Solutions . . . . . . . . . . . . . 100

3.6.3 A Formal Solution . . . . . . . . . . . . . . . 101

3.7 Energy Arguments . . . . . . . . . . . . . . . . . 102

3.8 Differentiation of Integrals . . . . . . . . . . . 106

3.9 Exercises . . . . . . . . . . . . . . . . . . 108

3.10 Projects . . . . . . . . . . . . . . . 113

4 Finite Difference Schemes for the Heat Equation 117

4.1 An Explicit Scheme . . . . . . . . . . . . . . . . . . 119

4.2 Fourier Analysis of the Numerical Solution . . . . . . . 122

4.2.1 Particular Solutions . . . . . . . . . . . . . . 123

4.2.2 Comparison of the Analytical and Discrete Solution 127

4.2.3 Stability Considerations . . . . . . . . . . . . 129

4.2.4 The Accuracy of the Approximation . . . . . . . 130

4.2.5 Summary of the Comparison . . . . . . . . . . . 131

4.3 Von Neumann's Stability Analysis . . . . . . . . . . . . 132

4.3.1 Particular Solutions: Continuous and Discrete . . . . 133

4.3.2 Examples . . . . . . . . . . . . . . . . . . . . . 134

4.3.3 A Nonlinear Problem . . . . . . . . . . . . . . . 137

4.4 An Implicit Scheme . . . . . . . . . . . . . . . . . . . 140

4.4 .1 Stability Analysis . . . . . . . . . . . . . . . . 143

4.5 Numerical Stability by Energy Arguments . . . . . . . 145

4.6 Exercises . . . . . . . . . . . . . . . . . . . . 148 
5.1 Separation of Variables . . . . . . . . . . . . . . . 160

5.2 Uniqueness and Energy Arguments . . . . . . . . . . . 163

5.3 A Finite Difference Approximation . . . . . . . . . . 165

5.3.1 Stability Analysis . . . . . . . . . . . . . 168

5.4 Exercises . . . . . . . . . . . . . . . 170

6 Maximum Principles 175

6.1 A Two-Point Boundary Value Problem . . . . . . . . . 175

6.2 The Linear Heat Equation . . . . . . . . . . . . . . . 178

6.2.1 The Continuous Case . . . . . . . . . . . 180

6.2 .2 Uniqueness and Stability . . . . . . . . . . 183

6.2.3 The Explicit Finite Difference Scheme . . . . . . . . 184

6.2.4 The Implicit Finite Difference Scheme . . . . . . . 186

6.3 The Nonlinear Heat Equation . . . . . . . . . . . . 188

6.3.1 The Continuous Case . . . . . . . . . . . . 189

6.3.2 An Explicit Finite Difference Scheme . . . . . . . . 190

6.4 Harmonic Functions . . . . . . . . . . . . . . 191

6.4.1 Maximum Principles for Harmonic Functions . . . . 193

6.5 Discrete Harmonic Functions . . . . . . . . . . . 195

6.6 Exercises . . . . . . . . . . . . . . . . 201

7 Poisson's Equation in Two Space Dimensions 209

7.1 Rectangular Domains . . . . . . . . . . . . . 209

7.2 Polar Coordinates . . . . . . . . . . . . . . 212

7.2 .1 The Disc . . . . . . . . . . . . . 213

7.2 .2 A Wedge . . . . . . . . . . . 216

7.2 .3 A Corner Singularity . . . . . . . . . . . . . 217

7.3 Applications of the Divergence Theorem . . . . . . . 218

7.4 The Mean Value Property for Harmonic Functions . . . . 222

7.5 A Finite Difference Approximation . . . . . . . . . . 225

7.5.1 The Five-Point Stencil . . . . . . . . . . . . 225

7.5 .2 An Error Estimate . . . . . . . . . . . . . . . . 228

7.6 Gaussian Elimination for General Systems . . . . . . . 230

7.6.1 Upper Triangular Systems . . . . . . . . . . . . . 230

7.6 .2 General Systems . . . . . . . . . . . . . . . 231

7.6 .3 Banded Systems . . . . . . . . . . . . . . 234

7.6.4 Positive Definite Systems . . . . . . . . . 236

7.7 Exercises $\ldots \ldots \ldots \ldots \ldots \ldots \ldots \ldots \ldots \ldots$

8 Orthogonality and General Fourier Series 245

8.1 The Full Fourier Series . . . . . . . . . . . . . . . . 246

8.1.1 Even and Odd Functions . . . . . . . . . 249

8.1.2 Differentiation of Fourier Series . . . . . . . . . 252

8.1.3 The Complex Form . . . . . . . . . . . . . . 255 
8.1.4 Changing the Scale . . . . . . . . . . . . 256

8.2 Boundary Value Problems and Orthogonal Functions . . . . 257

8.2.1 Other Boundary Conditions . . . . . . . . . . . 257

8.2.2 Sturm-Liouville Problems . . . . . . . . . . . . . . . 261

8.3 The Mean Square Distance . . . . . . . . . . . . . . . 264

8.4 General Fourier Series . . . . . . . . . . . . . . 267

8.5 A Poincaré Inequality . . . . . . . . . . . . . . 273

8.6 Exercises . . . . . . . . . . . . . 276

9 Convergence of Fourier Series 285

9.1 Different Notions of Convergence . . . . . . . . . . . . 285

9.2 Pointwise Convergence . . . . . . . . . . . . . . 290

9.3 Uniform Convergence . . . . . . . . . . . . . . 296

9.4 Mean Square Convergence . . . . . . . . . . . . . . 300

9.5 Smoothness and Decay of Fourier Coefficients . . . . . . . 302

9.6 Exercises . . . . . . . . . . . . . . 307

10 The Heat Equation Revisited 313

10.1 Compatibility Conditions . . . . . . . . . . . . 314

10.2 Fourier's Method: A Mathematical Justification . . . . . . . 319

10.2.1 The Smoothing Property . . . . . . . . . . . 319

10.2.2 The Differential Equation . . . . . . . . . . 321

10.2.3 The Initial Condition . . . . . . . . . . . . . . . 323

10.2.4 Smooth and Compatible Initial Functions . . . . . 325

10.3 Convergence of Finite Difference Solutions . . . . . . . . 327

10.4 Exercises . . . . . . . . . . . . . . . 331

11 Reaction-Diffusion Equations 337

11.1 The Logistic Model of Population Growth . . . . . . . . . 337

11.1.1 A Numerical Method for the Logistic Model . . . . . 339

11.2 Fisher's Equation . . . . . . . . . . . . . . . . . . . 340

11.3 A Finite Difference Scheme for Fisher's Equation . . . . . 342

11.4 An Invariant Region . . . . . . . . . . . . . . . . 343

11.5 The Asymptotic Solution . . . . . . . . . . . . 346

11.6 Energy Arguments . . . . . . . . . . . . . . . . . . . . 349

11.6.1 An Invariant Region . . . . . . . . . . . . . . . 350

11.6.2 Convergence Towards Equilibrium . . . . . . . . 351

11.6.3 Decay of Derivatives . . . . . . . . . . . . . 352

11.7 Blowup of Solutions . . . . . . . . . . . . . . 354

11.8 Exercises $\ldots \ldots \ldots \ldots \ldots$. . . . . . . . . . . 357

11.9 Projects . . . . . . . . . . . . . . . . . . 360

12 Applications of the Fourier Transform 365

12.1 The Fourier Transform . . . . . . . . . . . . . . 366

12.2 Properties of the Fourier Transform . . . . . . . . . 368 
12.3 The Inversion Formula . . . . . . . . . . . . . . . . 372

12.4 The Convolution ..................... 375

12.5 Partial Differential Equations . . . . . . . . . . . . . 377

12.5.1 The Heat Equation . . . . . . . . . . . . . . 377

12.5.2 Laplace's Equation in a Half-Plane . . . . . . . . . 380

12.6 Exercises ... . . . . . . . . . . . . 382

$\begin{array}{ll}\text { References } & \mathbf{3 8 5}\end{array}$

$\begin{array}{ll}\text { Index } & \mathbf{3 8 9}\end{array}$ 